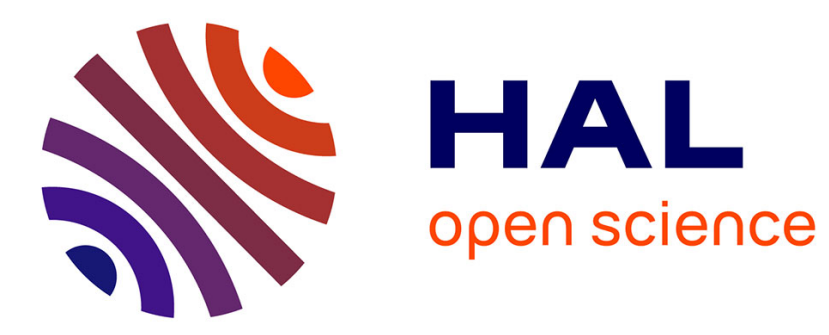

\title{
Parity-check matrix calculation for paraunitary oversampled DFT filter banks
}

Tania Karp, Michel Kieffer, Pierre Duhamel

\section{To cite this version:}

Tania Karp, Michel Kieffer, Pierre Duhamel. Parity-check matrix calculation for paraunitary oversampled DFT filter banks. IEEE Transactions on Signal Processing, 2008, 56 (10), pp.5277-5283. hal-00549122

\section{HAL Id: hal-00549122 \\ https://hal.science/hal-00549122}

Submitted on 21 Dec 2010

HAL is a multi-disciplinary open access archive for the deposit and dissemination of scientific research documents, whether they are published or not. The documents may come from teaching and research institutions in France or abroad, or from public or private research centers.
L'archive ouverte pluridisciplinaire HAL, est destinée au dépôt et à la diffusion de documents scientifiques de niveau recherche, publiés ou non, émanant des établissements d'enseignement et de recherche français ou étrangers, des laboratoires publics ou privés. 


\title{
Parity-Check Matrix Calculation for Paraunitary Oversampled DFT Filter Banks
}

Tanja Karp, Member, IEEE, Michel Kieffer, Member, IEEE, and Pierre Duhamel, Fellow, IEEE

\begin{abstract}
Oversampled filter banks, interpreted as error correction codes, were recently introduced in the literature. We here present an efficient calculation and implementation of the parity-check polynomial matrices for oversampled DFT filter banks. If desired, the calculation of the partity-check polynomials can be performed as part of the prototype filter design procedure. We compare our method to those previously presented in the literature.
\end{abstract}

EDICS Category: DSP-FILT, DSP-FAST

T. Karp is with the Department of Electrical and Computer Engineering, Texas Tech University P.O. Box 43102, Lubbock, TX, 79409, USA, e-mail: tanja.karp@ttu.edu.

M. Kieffer and P. Duhamel are with the Laboratoire des Signaux et Systèmes, CNRS - SUPELEC - Univ. de Paris-Sud, 91192 Gif-sur Yvette, France, e-mail: kieffer@1ss.supelec.fr, pierre.duhamel@1ss.supelec.fr

Manuscript received xx, 2008. 


\section{Parity-Check Matrix Calculation for}

\section{Paraunitary Oversampled DFT Filter Banks}

\section{INTRODUCTION}

During the last years, oversampled filter banks were proposed as an alternative to critically sampled ones since they provide a series of desirable properties, such as an improved robustness to quantization errors [1]-[3], an increased design flexibility [2], [4], [5], and the ability to recover signal erasures and transmission errors [6]-[9]. More recently, oversampled filter banks were also used in the context of joint source-channel coding and errorcorrection coding [8], [10]-[12]. In [10] the design of a parity-check polynomial matrix, which is implemented in parallel to the synthesis filter bank, is introduced. Its output, the so-called syndrom, is non-zero only in presence of subband signal errors, i.e. when the input of the synthesis filter bank differs from the output of the analysis filter bank, either due to quantization or due to transmission errors. The original subband signals can then be recovered using hypothesis testing [10], [11]. Oversampled filter banks can thus be interpreted as convolutive error-correction codes working in the real or complex domain. They provide an extension of redundant block transforms which had already been interpreted as error correction block codes [13]-[15] in the real or complex domain. The capability to correct impulse errors in the subbands despite the presence of Gaussian background noise based upon the received syndroms was demonstrated in [10], [11] and applied to robust image and video transmission in [8], [11], [16], [17].

Although the parity-check polynomial matrix design was derived for general oversampled filter banks in [8], [10], modulated filter banks [12], [18]-[22] were used in the applications presented since they have a reduced design complexity and can be implemented cost-efficiently exploiting fast transforms and sparse polyphase matrices [18], $[23]-[25]$.

We here present the calculation of a sparse parity-check polynomial matrix for oversampled DFT filter banks that exploits the efficient polyphase structure of these filter banks and results in a significantly reduced implementation cost for the partity check polynomial matrix when being compared to the general case. We show how our implementation relates to the general framework presented in [10], [11], such that the same hypothesis testing can be applied to detect impulse errors in Gaussian background noise. We further derive how the new sparse partity-check polynomial matrix is obtained either from a given prototype filter or directly calculated as part of the prototype filter design procedure.

Notations: Time domain signals are denoted by small letters and their $z$ transform by capital letters, $\mathcal{Z}\{x[n]\}=$ $X(z)^{1}$. Bold face letters are used for vectors and matrices, $\mathbf{x}^{T}$ is the transpose of $\mathbf{x}$, and $\mathbf{x}^{H}$ is the Hermitian of $\mathbf{x}$,

\footnotetext{
${ }^{1}$ For the sake of a more compact desciption in the $z$ domain, the existance of the $z$ transform of all signals is assumed, however, their existence is not a required.
} 
i.e its transpose with complex conjugate entries. $\mathbf{I}_{M}$ and $\mathbf{J}_{M}$ denote the identity matrix and counter identity matrix of size $M \times M$, respectively. $\mathbf{0}_{M \times N}$ is an all zero matrix of size $M \times N$. A matrix $\mathbf{U}(z)$ containing FIR polynomials is called unimodular if it has an inverse matrix $\mathbf{U}^{-1}(z)$ which is also $\operatorname{FIR} . \tilde{\mathbf{E}}(z)$ is the para-conjugate of $\mathbf{E}(z)$, W the $M \times M$ orthonormal DFT modulation matrix with entries $[\mathbf{W}]_{i, j}=W_{M}^{i j} / \sqrt{M}$, and $W_{M}=\exp (-j 2 \pi / M)$ being a complex rotation factor.

\section{Oversampled DFT Filter BANKS}

We consider oversampled DFT filter banks with $M$ subbands and a decimation factor of $N \leq M$, see Figure 1 . The greatest common divider of $M$ and $N$ is denoted by $b=\operatorname{gcd}(M, N)$, the least common multiple by $K=\operatorname{lcm}(M, N)$, and $J=M / b$. The causal FIR analysis filters $H_{k}(z)$ of length $L$ are obtained through complex modulation with a modulation phase offset $n_{a}$ from a lowpass prototype filter $e[n]$ :

$$
h_{k}[n]=\frac{1}{\sqrt{M}} e[n] \exp \left(j \frac{2 \pi k\left(n-n_{a}\right)}{M}\right)
$$

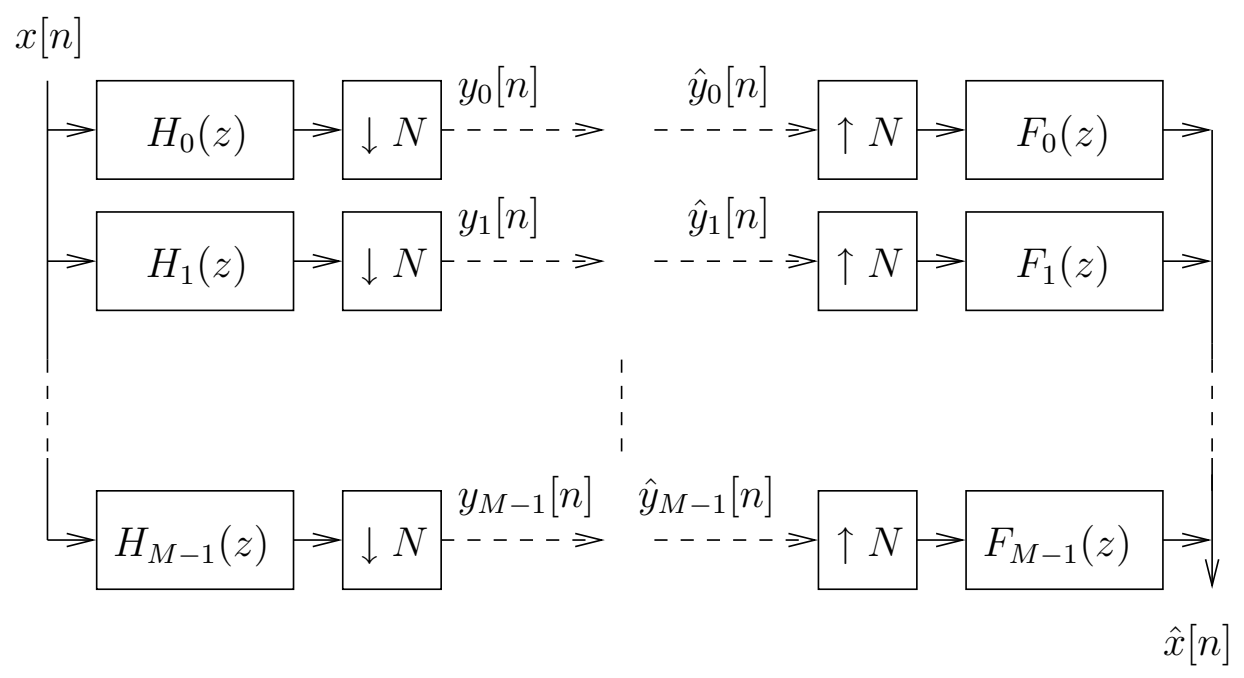

Fig. 1. Oversampled Filter Bank

The $M \times N$ polyphase matrix $\mathbf{H}_{p}(z)$, with $\left[\mathbf{H}_{p}(z)\right]_{k, \lambda}$ being the $\lambda$-th type-I polyphase component of $H_{k}(z)$, has the following efficient realization [18], [23], [25], [26]:

$$
\mathbf{H}_{p}(z)=\operatorname{diag}\left(1, W_{M}^{n_{a}}, \ldots, W_{M}^{n_{a}(M-1)}\right) \mathbf{W}_{M}^{H} \mathbf{E}(z)
$$

where $\mathbf{E}(z)$ is a sparse matrix with the following entries:

$$
\begin{gathered}
\quad[\mathbf{E}(z)]_{i, j}= \begin{cases}z^{-l} E_{j+l N}\left(z^{J}\right) & \text { if }(i-j) \bmod b=0 \\
0 & \text { otherwise }\end{cases} \\
i=0, \ldots, M-1, \quad j=0, \ldots N-1, \quad(j+l N) \bmod M=i
\end{gathered}
$$


and $E_{k}(z)$ is the $k$ th of $K$ type-I polyphase components of the analysis lowpass prototype filter $E(z)$.

For the filter bank to provide perfect reconstruction, i.e. $\hat{x}[n]=x[n]$ in Figure 1, the $N \times M$ synthesis polyphase matrix $\mathbf{F}_{p}(z)$ has to be the left inverse of the analysis polyphase matrix $\mathbf{H}_{p}(z)$ and can be expressed as [2]:

$$
\mathbf{F}_{p}(z)=\hat{\mathbf{F}}_{p}(z)+\mathbf{U}(z)\left[\mathbf{I}_{M}-\mathbf{H}_{p}(z) \hat{\mathbf{F}}_{p}(z)\right] \quad \text { with } \hat{\mathbf{F}}_{p}(z)=\left[\tilde{\mathbf{H}}_{p}(z) \mathbf{H}_{p}(z)\right]^{-1} \tilde{\mathbf{H}}_{p}(z)
$$

where $\hat{\mathbf{F}}_{p}(z)$ is the para-pseudoinverse of $\mathbf{H}_{p}(z)$. It was further shown in [3] that if the output of the analysis filter bank is corrupted by quantization noise which can be modelled as white uncorrelated noise with constant variance in all subbands, the synthesis filter bank with the lowest mean squared error (MSE) of the reconstructed signal is given by $\hat{\mathbf{F}}_{p}(z)$. The filter bank is paraunitary, if $\tilde{\mathbf{H}}_{p}(z) \mathbf{H}_{p}(z)=\mathbf{I}_{N}$ [24], thus if

$$
\hat{\mathbf{F}}_{p}(z)=\tilde{\mathbf{H}}_{p}(z)=\tilde{\mathbf{E}}(z) \mathbf{W}_{M} \operatorname{diag}\left(1, W_{M}^{-n_{a}}, \ldots, W_{M}^{-n_{a}(M-1)}\right)
$$

yielding in anti-causal FIR synthesis filters $F_{k}(z), k=0, \ldots, M-1$, with impulse responses

$$
f_{k}[-n]=h_{k}[n]=\frac{1}{\sqrt{M}} e[n] \exp \left(j \frac{2 \pi k\left(n-n_{a}\right)}{M}\right)
$$

The PR constraints for paraunitary oversampled DFT filter banks can now be expressed as [4], [21]

$$
\tilde{\mathbf{H}}_{p}(z) \mathbf{H}_{p}(z)=\tilde{\mathbf{E}}(z) \mathbf{E}(z)=\mathbf{I}_{N}
$$

and thanks to the sparse structure of $\mathbf{E}(z)$ they can be written as $b$ independent sets of PR constraints

$$
\begin{aligned}
& \tilde{\mathbf{E}}_{\ell}(z) \mathbf{E}_{\ell}(z)=\mathbf{I}_{N / b} \quad \text { with } \quad\left[\mathbf{E}_{\ell}(z)\right]_{k, j}=[\mathbf{E}(z)]_{\ell+k b, \ell+j b}, \\
& \ell=0, \ldots, b-1, \quad k=0, \ldots, J-1, \quad j=0, \ldots, N / b-1
\end{aligned}
$$

Since $\mathbf{E}_{\ell}(z)$ contains delayed $J$-fold upsampled polyphase components of the prototype filter with the same delay on each diagonal it can be expressed as [18], [21]

$$
\mathbf{E}_{\ell}(z)=\boldsymbol{\Lambda}_{1}(z) \mathbf{E}_{\ell \downarrow}\left(z^{J}\right) \boldsymbol{\Lambda}_{2}(z)
$$

with appropriate paraunitary diagonal matrices $\Lambda_{1}(z)$ and $\Lambda_{2}(z)$ containing monomials in $z$. The $J$ times downsampled version of the PR constraints now write

$$
\tilde{\mathbf{E}}_{\ell \downarrow}(z) \mathbf{E}_{\ell \downarrow}(z)=\mathbf{I}_{N / b}
$$

Note that the number of constraints in (10) equals $L_{e} b(N / b)^{2}=N^{2} / b$, where $L_{e}$ denotes the length of the polyphase filters $E_{\ell}(z)$, and is thus reduced by $J b$ when compared to (7).

Example: $M=8, N=6$

For a filter bank with $M=8$ subbands and a decimation factor of $N=6$ we have $b=\operatorname{gcd}(M, N)=2$, $K=\operatorname{lcm}(M, N)=24$, and $J=M / b=4$. The polyphase matrix $\mathbf{E}(z)$ can thus be split into $\mathbf{E}_{0}(z)$ and $\mathbf{E}_{1}(z)$ 
and using $\boldsymbol{\Lambda}_{1}(z)=\operatorname{diag}\left(1, z^{-1}, z^{-2}, z^{-3}\right)$ and $\boldsymbol{\Lambda}_{2}(z)=\operatorname{diag}\left(1, z, z^{2}\right)$ they can be factorized as:

$$
\begin{aligned}
& \mathbf{E}_{0}(z)=\left[\begin{array}{ccc}
E_{0}\left(z^{4}\right) & z^{-1} E_{8}\left(z^{4}\right) & z^{-2} E_{16}\left(z^{4}\right) \\
z^{-3} E_{18}\left(z^{4}\right) & E_{2}\left(z^{4}\right) & z^{-1} E_{10}\left(z^{4}\right) \\
z^{-2} E_{12}\left(z^{4}\right) & z^{-3} E_{20}\left(z^{4}\right) & E_{4}\left(z^{4}\right) \\
z^{-1} E_{6}\left(z^{4}\right) & z^{-2} E_{14}\left(z^{4}\right) & z^{-3} E_{22}\left(z^{4}\right)
\end{array}\right]=\Lambda_{1}(z) \underbrace{\left[\begin{array}{ccc}
E_{0}\left(z^{4}\right) & E_{8}\left(z^{4}\right) & E_{16}\left(z^{4}\right) \\
z^{-4} E_{18}\left(z^{4}\right) & E_{2}\left(z^{4}\right) & E_{10}\left(z^{4}\right) \\
z^{-4} E_{12}\left(z^{4}\right) & z^{-4} E_{20}\left(z^{4}\right) & E_{4}\left(z^{4}\right) \\
z^{-4} E_{6}\left(z^{4}\right) & z^{-4} E_{14}\left(z^{4}\right) & z^{-4} E_{22}\left(z^{4}\right)
\end{array}\right]}_{\mathbf{E}_{0 \downarrow}\left(z^{4}\right)} \\
& \mathbf{E}_{1}(z)=\left[\begin{array}{ccc}
E_{1}\left(z^{4}\right) & z^{-1} E_{9}\left(z^{4}\right) & z^{-2} E_{17}\left(z^{4}\right) \\
z^{-3} E_{19}\left(z^{4}\right) & E_{3}\left(z^{4}\right) & z^{-1} E_{11}\left(z^{4}\right) \\
z^{-2} E_{13}\left(z^{4}\right) & z^{-3} E_{21}\left(z^{4}\right) & E_{5}\left(z^{4}\right) \\
z^{-1} E_{7}\left(z^{4}\right) & z^{-2} E_{15}\left(z^{4}\right) & z^{-3} E_{23}\left(z^{4}\right)
\end{array}\right]=\boldsymbol{\Lambda}_{1}(z) \underbrace{\left[\begin{array}{ccc}
E_{1}\left(z^{4}\right) & E_{9}\left(z^{4}\right) & E_{17}\left(z^{4}\right) \\
z^{-4} E_{19}\left(z^{4}\right) & E_{3}\left(z^{4}\right) & E_{11}\left(z^{4}\right) \\
z^{-4} E_{13}\left(z^{4}\right) & z^{-4} E_{21}\left(z^{4}\right) & E_{5}\left(z^{4}\right) \\
z^{-4} E_{7}\left(z^{4}\right) & z^{-4} E_{15}\left(z^{4}\right) & z^{-4} E_{23}\left(z^{4}\right)
\end{array}\right]}_{\mathbf{E}_{1 \downarrow}\left(z^{4}\right)}
\end{aligned}
$$

Figure 2 shows the efficient polyphase realization of the paraunitary oversampled DFT filter bank where the vectorized input signal is denoted by $\mathbf{x}[n]=(x[n N], x[n N-1], \ldots, x[n N-N+1])^{T}$ and the subband signals are given by $\mathbf{y}[n]=\left(y_{0}[n], y_{1}[n], \ldots, y_{M-1}[n]\right)^{T}$. At the synthesis filter bank, the subband signals $\hat{\mathbf{y}}[n]=$ $\left(\hat{y}_{0}[n], \hat{y}_{1}[n], \ldots, \hat{y}_{M-1}[n]\right)^{T}$ are received. In absence of subband signal processing and transmission errors, $\hat{\mathbf{y}}[n]=$ $\mathbf{y}[n]$ holds true, otherwise $\hat{\mathbf{y}}[n]$ is a corrupted version of $\mathbf{y}[n]$ caused by subband coding and / or transmission errors.

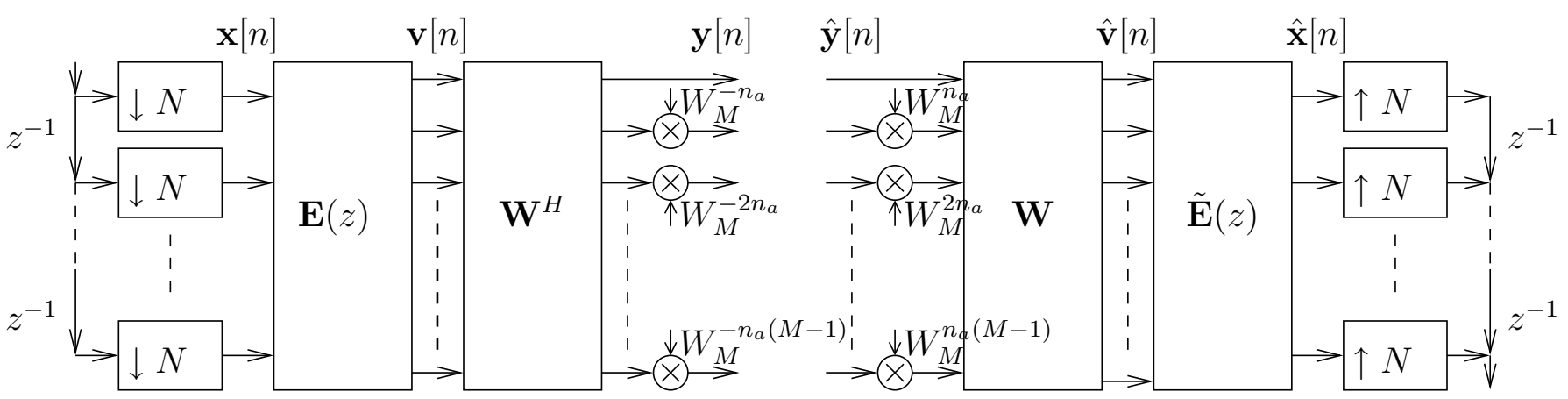

Fig. 2. Polyphase Realization of Oversampled DFT-Modulated Filter Bank

\section{Remarks:}

- There are a few minor differences between (2) and (5) and the form derived in [18], [26]:

- The DFT and IDFT modulation matrices at the analysis and synthesis filter bank are swapped due to a difference in modulation. The filters $H_{k}(z)$ in [18], [26] correspond to $H_{M-k}(z)$ in (1). Our scheme is identical to the one derived in [23], [24]. 
- The additional diagonal matrices $\operatorname{diag}\left(1, W_{M}^{n_{a}}, \ldots, W_{M}^{n_{a}(M-1)}\right)$ and $\operatorname{diag}\left(1, W_{M}^{-n_{a}}, \ldots, W_{M}^{-n_{a}(M-1)}\right)$ are due to the phase offset $n_{a}$ in our modulation which is required for the implementation of causal linear-phase analysis filters of arbitrary length $L$ when being be chosen as $(L-1) \bmod M$.

- To obtain causal synthesis filters, the synthesis filter impulse responses can be delayed by integer multiples of $N$ until causality is obtained. Depending on the length $L$ of the prototype filter, the introduction of leading zeros might be neccessary.

- An alternative implementation of the polyphase filters is described in [25].

\section{Sparse Parity Check Polynomials for Paraunitary Oversampled DFT Filter Banks}

The interpretation of oversampled filter banks as convolutive error correction codes was already presented in [8], [10]. A parity-check polynomial matrix $\mathbf{C}^{S B}(z)$ is implemented at the synthesis filter bank and calculates $M-N$ syndrom signals from the $M$ received subband signals $\hat{\mathbf{y}}[n]$. Since $\mathbf{C}^{S B}(z)$ satisfies

$$
\mathbf{C}^{S B}(z) \mathbf{H}_{p}(z)=\mathbf{0}
$$

the syndrom signals are identical to zero if $\hat{\mathbf{y}}[n]=\mathbf{y}[n]$, i.e. if neither subband signal processing such as quantization is performed nor transmission errors occur.

For the special case of paraunitary oversampled DFT filter banks, we here present an implementation of the parity-check polynimial matrix that extends the efficient polyphase realization of the filter bank to the parity-check polynomial matrix. Due to our assumption of causal analysis filters and anti-causal synthesis filters, we here assume that our parity check polynomial matrix also contains anti-causal filters. We factorize the parity check matrix in the same way as the synthesis polyphase matrix:

$$
\mathbf{C}^{S B}(z)=\tilde{\mathbf{C}}(z) \mathbf{W}^{H} \operatorname{diag}\left(1, W_{M}^{-n_{a}}, \ldots, W_{M}^{-n_{a}(M-1)}\right)
$$

Note that the complex rotations and the DFT are also performed by the synthesis filter bank and therefore present no additional implementation cost. The only additional implementation cost is the convolution of the DFT output signals $\hat{\mathbf{v}}[n]$ with the filters in $\tilde{\mathbf{C}}(z)$. Expressing $\mathbf{H}_{p}(z)$ according to (2), (13) simplifies to

$$
\tilde{\mathbf{C}}(z) \mathbf{E}(z)=\mathbf{0}_{(M-N) \times N}
$$

Since $\mathbf{E}(z)$ is sparse, see (3), the above constraints can again be expressed as $b$ sets of equations, yielding

$$
\begin{gathered}
\tilde{\mathbf{C}}_{\ell}^{(1)}(z) \mathbf{E}_{\ell}(z)=\mathbf{0}_{(M-N) \times N / b}, \quad \text { with } \quad\left[\tilde{\mathbf{C}}_{\ell}^{(1)}(z)\right]_{i, j}=[\tilde{\mathbf{C}}(z)]_{i, \ell+j b}, \\
\ell=0, \ldots, b-1, \quad i=0, \ldots,(M-N)-1, \quad j=0, \ldots, J-1
\end{gathered}
$$

Since all $\mathbf{E}_{\ell}(z)$ have to be of rank $N / b$ to sarisfy (8), their null space is of dimentionality $(M-N) / b$, and thus only $(M-N) / b$ of the $M-N$ rows of $\tilde{\mathbf{C}}_{\ell}^{(1)}(z)$ can be linearly independent. Without loss of generality, we choose them as

$$
\begin{gathered}
\tilde{\mathbf{C}}_{\ell}(z) \mathbf{E}_{\ell}(z)=\mathbf{0}_{(M-N) / b \times N / b}, \quad \text { with } \quad\left[\tilde{\mathbf{C}}_{\ell}(z)\right]_{i, j}=[\tilde{\mathbf{C}}(z)]_{\ell+i b, \ell+j b}, \\
\ell=0, \ldots, b-1, \quad i=0, \ldots,(M-N) / b-1, \quad j=0, \ldots, J-1
\end{gathered}
$$


resulting in parity-check polynomial matrices $\tilde{\mathbf{C}}_{\ell}(z)$ of size $(M-N) / b \times J$. Expressing $\tilde{\mathbf{C}}_{\ell}(z)$ as

$$
\tilde{\mathbf{C}}_{\ell}(z)=\tilde{\mathbf{C}}_{\ell \downarrow}\left(z^{J}\right) \tilde{\boldsymbol{\Lambda}}_{1}(z)
$$

and using (9) we finally obtain a downsampled version of (17) as

$$
\tilde{\mathbf{C}}_{\ell \downarrow}(z) \mathbf{E}_{\ell \downarrow}(z)=\mathbf{0}_{(M-N) / b \times N / b},
$$

Example: $M=8, N=6$

For our example with $M=8$ subbands and a decimation factor of $N=6$, (19) writes:





Note that we have split the calculation of the $M-N$ syndroms into the parallel implementation of $b$ parity-check polynomial matrices $\tilde{\mathbf{C}}_{l}(z)$ of size $(M-N) / b \times J$ that take the vectors $\hat{\mathbf{v}}_{\ell}[n]=\left[\hat{v}_{\ell}[n], \hat{v}_{\ell+b}[n], \ldots, \hat{v}_{\ell+(J-1) b}[n]\right]^{T}$ as input signals to calculate the syndrom vectors $\mathbf{s}_{\ell}[n]=\left[s_{\ell}[n], s_{\ell+b}[n], \ldots, s_{\ell+M-N-b}[n]\right]^{T}$. The resulting scheme is shown in Figure 3. Since in addition all $\tilde{\mathbf{C}}_{l}(z)$ contain shifted polynomials in $z^{J}$, the implementation cost is further reduced.


Fig. 3. General parity-check polynomial matrix and new sparse parity-check polynomial submatrices for modulated DFT filter banks

Since the here described sparse parity check polynomial matrices relate to the ones proposed in [8], [10] through (14), the hypothesis tests described therein to detect impulse errors in Gaussian background noise can be applied in the same way. 


\section{Computation of Parity-Check Polynomial Matrix}

For the case of general oversampled filter banks, it was proposed in [10], [27] to obtain the parity-check polynomial matrix $\mathbf{C}^{S B}(z)$ from (14) via Smith-McMillan decomposition [24] of the analysis polyphase matrix $\mathbf{H}_{p}(z)$ :

$$
\mathbf{H}_{p}(z)=\mathbf{U}(z)\left[\begin{array}{c}
\boldsymbol{\Lambda}(z) \\
\mathbf{0}_{(M-N) \times N}
\end{array}\right] \mathbf{W}(z), \quad \mathbf{C}^{S B}(z)=\left[\begin{array}{ll}
\mathbf{0}_{M-N, N} & \mathbf{I}_{M-N}
\end{array}\right] \mathbf{U}^{-1}(z)
$$

where $\mathbf{U}(z)$ and $\mathbf{W}(z)$ are unimodular $M \times M$ and $N \times N$ matrices, respectively, and $\boldsymbol{\Lambda}(z)$ is an $N \times N$ diagonal matrix. While we could apply this approach to solve (19), which is of reduced dimention and contains lower order polynomials, it is well known that it suffers from numerical stability problems [10], [28]. Also, U(z) and W(z) are not unique and the polynomial degrees can become unnessarily high, resulting in an increased complexity of the hypothesis tests described in [10], [11] to detect and correct impulse errors. To overcome this problem, it was suggested in [10] to calculate the coefficients of $\mathbf{C}^{S B}(z)$ in the time domain from a numerically stable QR decomposition [29] of a column-permuted Sylvester matrix obtained from $\mathbf{H}_{p}(z)$. For oversampled DFT filter banks, this approach can be directly applied to (19), which in the time domain writes:

$$
\left[\begin{array}{llll}
\mathbf{C}_{\ell \downarrow}^{L_{c}-1} & \mathbf{C}_{\ell \downarrow}^{L_{c}-2} & \ldots & \mathbf{C}_{\ell \downarrow}^{0}
\end{array}\right] \underbrace{\left[\begin{array}{cccccccc}
\mathbf{E}_{\ell \downarrow}^{L_{e}-1} & \mathbf{E}_{\ell \downarrow}^{L_{e}-2} & \ldots & \mathbf{E}_{\ell \downarrow}^{0} & \mathbf{0}_{J \times N / b} & \ldots & \mathbf{0}_{J \times N / b} \\
\mathbf{0}_{J \times N / b} & \mathbf{E}_{\ell \downarrow}^{L_{e}-1} & \mathbf{E}_{\ell \downarrow}^{L_{e}-2} & \ldots & \mathbf{E}_{\ell \downarrow}^{0} & \ddots & \vdots \\
\vdots & \ddots & \ddots & & & \ddots & \mathbf{0}_{J \times N / b} \\
\mathbf{0}_{J \times N / b} & \ldots & \mathbf{0}_{J \times N / b} & \mathbf{E}_{\ell \downarrow}^{L_{e}-1} & \mathbf{E}_{\ell \downarrow}^{L_{e}-2} & \ldots & \mathbf{E}_{\ell \downarrow}^{0}
\end{array}\right]}_{\mathbf{S}_{B_{\ell}}}=\left[\begin{array}{c}
\mathbf{0}_{(M-N) / b \times N / b} \\
\vdots \\
\vdots \\
\mathbf{0}_{(M-N) / b \times N / b}
\end{array}\right]
$$

with $\mathbf{C}_{\ell \downarrow}(z)=\sum_{i=0}^{L_{c}-1} \mathbf{C}_{\ell \downarrow}^{i} z^{-i}$ and $\mathbf{E}_{\ell \downarrow}(z)=\sum_{i=0}^{L_{e}-1} \mathbf{E}_{\ell \downarrow}^{i} z^{-i}$ and $L_{e}=\lceil L / K\rceil$. A solution to the upper equation only exists if the $L_{c} M \times\left(L_{e}-1+q\right) N$ Sylvester matrix $\mathbf{S}_{B_{\ell}}$ has a rank that is at least $(M-N) / b$ smaller than its number of rows. A sufficient condition herefore is that $\mathbf{S}_{B_{\ell}}$ is tall with at least $(M-N) / b$ more rows than columns, i.e. if $L_{c}-1 \geq\left(L_{e}-1\right) \frac{N}{M-N}$. We perform a QR decomposition of $\mathbf{S}_{B_{\ell}}=\mathbf{Q}_{\ell} \mathbf{F}_{\mathbf{p}_{\ell}}$ and the coefficients of $\left[\begin{array}{llll}\mathbf{C}_{\ell \downarrow}^{L_{c}-1} & \mathbf{C}_{\ell \downarrow}^{L_{c}-2} & \ldots & \mathbf{C}_{\ell \downarrow}^{0}\end{array}\right]$ are obtained as the last $(M-N) / b$ rows of $\mathbf{Q}_{\ell}$. Numerical stabilization can be improved by performing column permutations of $\mathbf{S}_{B_{\ell}}$ as described in [29].

So far, we have assumed that the parity-check polynomial matrices $\tilde{\mathbf{C}}_{\ell}(z)$ are calculated for an existing prototype filter $E(z)$. If, however, the prototype filter still needs to be designed, then the calculation of the partity-check polynomials can be easily included into the design procedure. For this purpose, we combine (10) and (19) to

$$
\left[\begin{array}{l}
\tilde{\mathbf{E}}_{\ell \downarrow}(z) \\
\tilde{\mathbf{C}}_{\ell \downarrow}(z)
\end{array}\right]\left[\begin{array}{ll}
\mathbf{E}_{\ell \downarrow}(z) & \mathbf{C}_{\ell \downarrow}(z)
\end{array}\right]=\mathbf{I}_{J}, \quad \ell=0, \ldots, b-1
$$

such that we obtain square matrices. Note that we have introduced $((M-N) / b)^{2}$ additional constraints through $\tilde{\mathbf{C}}_{\ell \downarrow}(z) \mathbf{C}_{\ell \downarrow}(z)=\mathbf{I}_{(M-N) / b \times(M-N) / b}$. These constraints ensure that pairwise uncorrelated white noise in the 
subband signals with constant variance, as it is often assumed as a model for quantization noise, causes syndroms which are also pairwise uncorrelated, white and have the same variance. Thus, for the detection of impulse noise in Gaussian background noise, they provide additional information on the statistical properties of the syndroms in absense of impulse errors. (24) was actually already proposed in [21] to facilitate the prototype design procedure for oversampled modulated filter banks. There, the authors factorize $\left[\begin{array}{lll}\mathbf{E}_{\ell \downarrow}(z) & \mathbf{C}_{\ell \downarrow}(z)\end{array}\right]$ into paraunitary lattices

$$
\begin{gathered}
{\left[\begin{array}{cc}
\mathbf{E}_{\ell \downarrow}(z) & \mathbf{C}_{\ell \downarrow}(z)
\end{array}\right]=\prod_{i=0}^{L_{e}-1} \mathbf{v}_{\ell, i}(z) \prod_{j=1}^{J-1} \mathbf{U}_{\ell, j},} \\
\mathbf{V}_{\ell, i}(z)=\mathbf{I}_{J}-\mathbf{v}_{\ell, i} \mathbf{v}_{\ell, i}^{T}+z^{-1} \mathbf{v}_{\ell, i} \mathbf{v}_{\ell, i}^{T}, \quad \mathbf{U}_{\ell, j}=\mathbf{I}_{J}-2 \mathbf{u}_{\ell, j} \mathbf{u}_{\ell, j}^{T},
\end{gathered}
$$

and then optimize the entries of $\mathbf{v}_{\ell, i}$ and $\mathbf{u}_{\ell, j}$ according to some cost function, such as the stopband energy of the prototype filter. The resulting parity-check polynomial matrices are of the same order $L_{e}-1$ as the polyphase filters. However, the authors did not realize the significance of $\mathbf{C}_{\ell, \downarrow}(z)$ and once the unconstrained optimization is completed, they discard these coefficients. The reader is referred to [21] for more details on the design procedure and optimization.

The propotype filter and parity-check polynomial matrices can also be designed by extending the quadraticconstrained least-squares (QCLS) optimization proposed in [30] to oversampled filter banks with perfect reconstruction. With $\mathbf{e}=\left[e_{0}, e_{1}, \ldots, e_{L-1}\right]^{T}$ being the vector of the prototype filter coefficients and $\mathbf{c}=\left[c_{0}, c_{1}, \ldots, c_{L_{c}-1}\right]$ a vector gathering all coefficients of the parity-check polynomial matrices, one then optimizes

$$
\begin{aligned}
& \arg \min _{\mathbf{e}}\left(\mathbf{e}^{T} \mathbf{P} \mathbf{e}\right) \quad \text { subject to } \\
& {\left[\begin{array}{cccc}
\left(\mathbf{E}_{\ell \downarrow}^{L_{e}-1}\right)^{H} & \left(\mathbf{E}_{\ell \downarrow}^{L_{e}-2}\right)^{H} & \ldots & \left(\mathbf{E}_{\ell \downarrow}^{0}\right)^{H} \\
\left(\mathbf{C}_{\ell \downarrow}^{L_{e}-1}\right)^{H} & \left(\mathbf{C}_{\ell \downarrow}^{L_{e}-2}\right)^{H} \ldots & & \left(\mathbf{C}_{\ell \downarrow}^{0}\right)^{H}
\end{array}\right] \mathbf{Q}_{\ell}^{k}\left[\begin{array}{cc}
\mathbf{E}_{\ell \downarrow}^{0} & \mathbf{C}_{\ell \downarrow}^{0} \\
\vdots & \\
\mathbf{E}_{\ell \downarrow}^{L_{e}-2} & \mathbf{C}_{\ell \downarrow}^{L_{e}-2} \\
\mathbf{E}_{\ell \downarrow}^{L_{e}-1} & \mathbf{C}_{\ell \downarrow}^{L_{e}-1}
\end{array}\right]=\left\{\begin{array}{cc}
\mathbf{I}_{J} & \text { if } k=0 \\
\mathbf{0}_{J \times J} & \text { if } k=1,2, \ldots, L_{e}-1
\end{array}\right.}
\end{aligned}
$$

where $\mathbf{P}$ is a real, symmetric, and positive-definite matrix, depending on the stopband cutoff frequency and the filter length $L$, see [31], and

$$
\left[\mathbf{Q}_{\ell}^{k}\right]_{i, j}=\left\{\begin{array}{ll}
1 & \text { if }(j-i)=k J \\
\mathbf{0} & \text { otherwise }
\end{array} \quad, \quad i, j=0, \ldots,\left(L_{e}-1\right) J-1\right.
$$

\section{Design EXAmples}

\section{CONCLUSION}

The conclusion goes here.

\section{ACKNOWLEDGMENT}

The authors would like to thank... 


\section{REFERENCES}

[1] V. Goyal, M. Vetterli, and T. T. Nguyen, "Quantized overcomplete expansions in $r^{n}$ : Analysis, synthesis, and algorithms," IEEE Trans. on Information Theory, vol. 44, no. 1, pp. 16 - 31, January 1998.

[2] H. Bölcskei, F. Hlawatsch, and H. G. Feichtinger, "Frame-theoretic analysis of oversampled filter banks," IEEE Trans. on Signal Processing, vol. 46, no. 12, pp. 3256-3268, December 1998.

[3] H. Bölcskei and F. Hlawatsch, "Noise reduction in oversampled filter banks using predictive quantization," IEEE Trans. on Information Theory, vol. 47, no. 1, pp. 155-172, January 2001.

[4] Z. Cvetković and M. Vetterli, “Oversampled filter banks," IEEE Trans. on Signal Processing, vol. 46, no. 5, pp. 1245 - 1255 , May 1998.

[5] T. Tanaka and Y. Yamashita, "The generalized lapped pseudo-biorthogonal transform," in IEEE International Conference on Acoustics, Speech, and Signal Processing (ICASSP), vol. II, Orlando, FL, 2002, pp. 1273-1276.

[6] V. K. Goyal, J. Kovacević, and J. A. Kelner, "Quantized frame expansions with erasures," Journal of Appl. and Comput. Harmonic Analysis, vol. 10, no. 3, pp. 203-233, 2001.

[7] J. Kovacević, P. L. Dragotti, and V. K. Goyal, "Filter bank frame expansions with erasures," IEEE Trans. on Information Theory, vol. 48, no. 6, pp. 1439-1450, 2002.

[8] S. Marinkovic and C. Guillemot, "Joint source-channel coding based on cosine-modulated filter banks for erasure-resilient signal transmission," EURASIP Journal on Applied Signal Processing, vol. 2005, no. Article ID 82023, pp. 510-524, 2005.

[9] Y. Sriraja and T. Karp, "A packetized SPIHT algorithm with overcomplete wavelet coefficients for increased robustness," EURASIP Journal on Applied Signal Processing, pp. 1 - 8, 2006.

[10] F. Labeau, J.-C. Chiang, M. Kieffer, P. Duhamel, L. Vandendorpe, and B. Macq, "Oversampled filter banks as error correction codes: Theory and impulse noise correction,” IEEE Trans. on Signal Processing, vol. 53, no. 12, pp. 4619 - 4630, December 2005.

[11] S. Marinkovic and C. Guillemot, "Joint source-channel coding by means of an oversampled filter bank code," EURASIP Journal on Applied Signal Processing, vol. 2006, no. Article ID 82023, pp. 1-12, 2006.

[12] S. Weiss, S. Redif, T. Cooper, C. Liu, P. D. Baxter, and J. G. McWhirter, "Paraunitary oversampled filter bank design for channel coding," EURASIP Journal on Applied Signal Processing, vol. 2006, no. Article ID 31346, pp. 1-10, 2006.

[13] G. Rath and C. Guillemot, "Frame-theoretic analysis of dft codes with erasures," IEEE Transactions on Signal Processing, vol. 52, no. 2, pp. $447-460$, feb 2004.

[14] A. Gabay, M. Kieffer, and P. Duhamel, "Joint source-channel coding using real BCH codes for robust image transmission," IEEE Trans. on Image Processing, vol. 16, no. 6, pp. 1568-1583, June 2007.

[15] W. Henkel, Applied Algebra, Algebraic Algorithms and Error-Correcting Codes, ser. Lecture Notes in Computer Science. Springer Berlin / Heidelberg, 1989, vol. 357/1989, ch. Multiple error correction with Analog Codes, pp. 239 - 249.

[16] J. C. Chiang, M. Kieffer, and P. Duhamel, "Robust image transmission using oversampled filterbank," in European Signal Processing Conference (EUSIPCO), 2004, pp. 1325 - 1328.

[17] J. C. Chiang, C. M. Lee, M. Kieffer, and P. Duhamel, "Robust video transmission over mixed IP -- wireless channels using motioncompensated oversampled filterbanks," in IEEE International Conference on Acoustics, Speech, and Signal Processing (ICASSP), 2006, pp. $1-4$.

[18] Z. Cvetković and M. Vetterli, "Tight weyl-heisenberg frames in $\ell^{2}(z)$," IEEE Trans. on Signal Processing, vol. 46, no. 5, pp. 1245 1255, May 1998.

[19] J. Kliewer and A. Mertins, "Oversampled cosine-modulated filter banks with arbitrary system delay," IEEE Trans. on Signal Processing, vol. 46, no. 4, pp. 941 - 955, April 1998.

[20] H. Bölcskei and F. Hlawatsch, "Oversampled cosine modulated filter banks with perfect reconstruction," IEEE Trans. on Circuits and Systems II, vol. 45, no. 8, pp. 1057-1071, August 1998.

[21] K. Eneman and M. Moonen, "DFT modulated filter bank design for oversampled subband systems," Signal Processing, vol. 81, pp. 1947 $-1973,2001$.

[22] C. Siclet, P. Siohan, and D. Pichon, "Perfect reconstruction conditions and design of oversampled dft-modulated transmultiplexers," EURASIP Journal on Applied Signal Processing, pp. 1-14, 2006.

[23] L. R. Rabiner and R. E. Crochiere, Multirate Signal Processing. Prentice Hall, 1993.

[24] P. P. Vaidyanathan, Multirate Systems And Filter Banks. Prentice Hall, 1993. 
[25] A. Weiss and R. W. Steward, "Fast implementation of oversampled modulated filter banks," IEEE Electronic Letters, vol. 36, no. 17, pp. 1502 - 1503, August 2000.

[26] Z. Cvetković, "Oversampled modulated filter banks and tight Gabor frames $l^{2}(z)$," in IEEE International Conference on Acoustics, Speech, and Signal Processing (ICASSP), 1995, pp. 1456 - 1459.

[27] F. Labeau, "Errors and losses in image transmission: Error-correcting and recovering schemes," Ph.D. dissertation, Université Catholique de Louvain, Louvain-la-Neuve, 2000.

[28] T. Karp and G. Schuller, "Joint transmitter / receiver design for multicarrier data transmission with low latency time," in IEEE International Conference on Acoustics, Speech, and Signal Processing (ICASSP), vol. 4, Salt Lake City, UT, USA, May 2001, pp. 2401 - 2404.

[29] D. Henrion and M. Sebek, "Reliable numerical methods for polynomial matrix triangularization," IEEE Trans. on Automatic Control, vol. 44, no. 3, pp. 497 - 508, March 1999.

[30] T. Q. Nguyen, "Digital filter bank design quadratic-constrained formulation," IEEE Trans. on Signal Processing, vol. 43, no. 9, pp. 2103 - 2108, September 1995.

[31] A. Tkacenko, P. P. Vaidyanathan, and T. Q. Nguyen, "On the eigenfilter design method and its applications: a tutorial," IEEE Trans. on Circuits and Systems II, vol. 50, no. 9, pp. 497 - 517, September 2003. 